\title{
STEEL BRIDGE RUST IDENTIFICATION USING MULTI-RESOLUTION PATTERN CLASSIFICATION
}

\author{
Po-Han Chen ${ }^{1}$, Yuh-Chin Chang ${ }^{2}$, and Luh-Maan Chang ${ }^{3}$
}

\author{
${ }^{1}$ Ph.D. Candidate, Div. of Constr. Eng. and Mgmt., School of Civil Eng., \\ Purdue Univ., West Lafayette, IN 47907, USA.E-mail: pohan@purdue.edu \\ ${ }^{2}$ Ph.D., School of Electrical and Computer Engineering, Purdue University, \\ West Lafayette, IN 47907, USA \\ ${ }^{3}$ Associate Professor, Div. of Constr. Eng. and Mgmt., School of Civil Eng., \\ Purdue Univ., West Lafayette, IN 47907, USA.
}

\begin{abstract}
Multi-resolution pattern classification (MPC) is an image segmentation technique that extracts features from an image and segments the image into several featured areas. Two resolution levels are contained in the MPC approach, the fine resolution level and the coarse resolution level. Pre-selected features, such as means and variances, will be computed, and then clustered using a linear or nonlinear classifier. This paper introduces the MPC approach to process steel bridge painting images with the hope of obtaining better segmentation results on rust identification.
\end{abstract}

Keywords: multi-resolution pattern classification (MPC), spatial gray level dependence method (SGLDM), gray level difference method (GLDM), clustering, homomorphic filtering.

\section{INTRODUCTION}

A fundamental and essential step to describe an image is to extract the underlying features of the scene. Typical applications include medical imagery, texture segmentation, remote sensing, geology, and robotics. A couple of papers have widely surveyed different techniques in feature selection and image segmentation [1,2,3]. Although a broad variety of methodologies have been applied to image segmentation problems, focuses are still placed on the development of feature-based approaches, which are the main concerns of this paper. Several algorithms have been investigated and compared in terms of how much image information can be contained in the proposed statistics. The results indicated that the spatial gray level dependence method (SGLDM) is the most powerful algorithm among the tested algorithms. In addition, the gray level difference method (GLDM) also works well on feature extraction in the spatial domain. Thus, the statistics used in both SGLDM and GLDM were adopted as the fundamental image features of the MPC method [4].

Similar to the region-based algorithms, the chosen statistics were computed and stored region by region, and then each region was classified according to the obtained statistics. Apparently, the multiresolution idea can be utilized to cope with this fashion $[5,6,7]$. At the measurement level (or the fine resolution level), an image is divided into blocks and each block is corresponding to one pixel at the coarse resolution level, where each dimension represents one category in the feature space. The pixels at the coarse resolution level will be classified using the clustering techniques from the pattern recognition field. After computing the pre-chosen features for each block at the fine resolution level, each pixel at the coarse resolution level, which is associated with one single block at the measurement level, will be mapped into a high dimensional space where every dimension stands for one chosen feature. Typical linear or nonlinear classifiers or clustering algorithms are then used to conduct class separation in the high dimensional feature space.

Two major concerns are involved in discriminating the features in a high dimensional space. First, the types and number of samples in the virtual feature space should be identified. Second, an appropriate classifier should be selected for feature clustering. The first concern depends on the preselected statistics and the size of partition blocks. The dimension of samples equals to the number of features. In general, four to eight features are adequate for segmentation purposes. More partitions in an image lead to a larger sample size. For a fixed number of features, more samples imply longer computation time. Obviously, these limitations somewhat make a trade-off between the efficiency and the performance necessary. As for the second concern, the determination of classifiers has a strong influence on the result of segmentation. The Bayes error is usually considered as the best criterion for feature evaluation, and a posteriori probability functions of samples are the ideal features. Unfortunately, a posteriori probability functions of 
samples are practically hard to obtain. Even using nonparametric density estimation techniques, the resulting Bayes errors usually have severe biases and variances [8]. Therefore, some other criteria are needed for feature classification. Generally, a linear classifier is effective and suitable for feature discrimination. Nonlinear classifiers may be expected to improve the segmentation performance, with the expense of more computational efforts.

\section{STATISTICAL FEATURES}

The statistical features adopted in the multiresolution pattern classification (MPC) method were originated from the spatial gray level dependence method (SGLDM) and the gray level difference method (GLDM) [3,9].

\subsection{Features of SGLDM}

The spatial gray level dependence method is based on the estimation of the second-order joint conditional probability density function $p(i, j \mid d, \theta)$, where $\theta=0^{\circ}, 45^{\circ}, 90^{\circ}, 135^{\circ}, 180^{\circ}, 225^{\circ}, 270^{\circ}$, and $315^{\circ}$. $p(i, j \mid d, \theta)$ represents the probability of going from gray level $i$ to gray level $j$, where $d$ is the intersample distance and the angle $\theta$ represents the direction. Each of the estimated second-order joint conditional density functions can be written in the matrix form:

$$
\Phi(d, \theta)=[p(i, j \mid d, \theta)] .
$$

The estimated probability matrices are assumed to have the following properties:

$$
\begin{gathered}
\Phi\left(d, 0^{\circ}\right)=\Phi^{T}\left(d, 180^{\circ}\right), \\
\Phi\left(d, 45^{\circ}\right)=\Phi^{T}\left(d, 225^{\circ}\right), \\
\Phi\left(d, 90^{\circ}\right)=\Phi^{T}\left(d, 270^{\circ}\right), \\
\Phi\left(d, 135^{\circ}\right)=\Phi^{T}\left(d, 315^{\circ}\right),
\end{gathered}
$$

where $\Phi^{T}(d, \theta)$ denotes the transpose of the matrix $\Phi(d, \theta)$. The main spatial gray level dependence matrices, $S_{0}(d), S_{45}(d), S_{90}(d)$, and $S_{135}(d)$ can then be defined as:

$$
\begin{aligned}
& S_{0}=\frac{1}{2}\left[\Phi\left(d, 0^{\circ}\right)+\Phi\left(d, 180^{\circ}\right)\right]=\frac{1}{2}\left[\Phi\left(d, 0^{\circ}\right)+\Phi^{T}\left(d, 0^{o}\right)\right] \\
& S_{45}=\frac{1}{2}\left[\Phi\left(d, 45^{\circ}\right)+\Phi\left(d, 225^{\circ}\right)\right]=\frac{1}{2}\left[\Phi\left(d, 45^{\circ}\right)+\Phi^{T}\left(d, 45^{\circ}\right)\right] \\
& S_{90}=\frac{1}{2}\left[\Phi\left(d, 90^{\circ}\right)+\Phi\left(d, 270^{\circ}\right)\right]=\frac{1}{2}\left[\Phi\left(d, 90^{\circ}\right)+\Phi^{T}\left(d, 90^{\circ}\right)\right] \\
& S_{135}=\frac{1}{2}\left[\Phi\left(d, 135^{\circ}\right)+\Phi\left(d, 315^{\circ}\right)\right]=\frac{1}{2}\left[\Phi\left(d, 135^{\circ}\right)+\Phi^{T}\left(d, 135^{\circ}\right)\right]
\end{aligned}
$$

From these matrices, four useful features can be defined as follows:

1. Contrast:

$$
C O N=\sum_{i=0}^{N-1} \sum_{j=0}^{N-1}(i-j)^{2} S_{\theta}(i, j \mid d)
$$

2. Angular Second Moment:

3. Entropy:

$$
A S M=\sum_{i=0}^{N-1} \sum_{j=0}^{N-1} S_{\theta}(i, j \mid d)^{2}
$$

$$
E N T=-\sum_{i=0}^{N-1} \sum_{j=0}^{N-1} S_{\theta}(i, j \mid d) \log S_{\theta}(i, j \mid d)
$$

\section{Correlation:}

$$
C O R=\frac{\sum_{i=0}^{N-1} \sum_{j=0}^{N-1}\left(i-\mu_{x}\right)\left(j-\mu_{y}\right) S_{\theta}(i, j \mid d)}{\sigma_{x} \sigma_{y}}
$$

where

$$
\begin{gathered}
\mu_{x}=\sum_{i=0}^{N-1} \sum_{j=0}^{N-1} i S_{\theta}(i, j \mid d) \\
\mu_{y}=\sum_{i=0}^{N-1} \sum_{j=0}^{N-1} j S_{\theta}(i, j \mid d) \\
\sigma_{x}=\sum_{i=0}^{N-1} \sum_{j=0}^{N-1}\left(i-\mu_{x}\right)^{2} S_{\theta}(i, j \mid d) \\
\sigma_{y}=\sum_{i=0}^{N-1} \sum_{j=0}^{N-1}\left(i-\mu_{y}\right)^{2} S_{\theta}(i, j \mid d)
\end{gathered}
$$

Furthermore, $S_{\theta}(i, j \mid d)$ is the $(i, j)^{\text {th }}$ element of $S_{\theta}(d)$ and $N$ is the number of gray levels in the image. The number of gray levels is usually smaller than 256 in image segmentation so as to reduce the computation time. According to past experiences, 32 gray levels are adequate for binary segmentation.

\subsection{Features of GLDM}

In order to estimate the underlying statistics of the gray level difference method, $g_{\delta}(m, n)=\mid g(m, n)-$ $g(m+\Delta m, n+\Delta n) \mid$ is defined, where $\delta$ is the displacement of $(\Delta m, \Delta n)$ with respect to the pixel $(m, n)$. Let $p(i \mid \delta)$ represent the probability density function associated with the possible values of $g_{\delta}$ :

$$
p(i \mid \delta)=\mathrm{P}\left(g_{\delta}(m, n)=i\right)
$$

Consider only four special cases of vector $\delta=(\Delta m$, $\Delta n)$. They are $(0, d),(0,-d),(d, 0)$, and $(-d, 0)$, respectively, where $d$ refers to the inter-sample spacing distance. From each of these density functions, four statistics are defined as follows:

1. Contrast:

$$
C O N=\sum_{i=0}^{N-1} i^{2} p(i \mid \delta)
$$

2. Angular Second Moment:

$$
A S M=\sum_{i=0}^{N-1}[p(i \mid \delta)]^{2}
$$

3. Entropy:

$$
E N T=-\sum_{i=0}^{N-1} p(i \mid \delta) \log p(i \mid \delta)
$$

4. Mean:

$$
M E A N=\sum_{i=0}^{N-1} i p(i \mid \delta)
$$

Since each feature of SGLDM depends on the parameter $\theta$ that has four major angles, $0^{\circ}, 45^{\circ}, 90^{\circ}$, and $135^{\circ}$, there are actually 16 statistics, 4 for each feature. Similarly, the statistics of GLDM are determined by the direction of the displacement vector $\delta$. Thus, there are four possibilities for each feature. Evidently, 32 statistics at most can be measured by evaluating all the features of SGLDM and GLDM. 


\section{PROBLEM FORMULATION}

The primary concept of this technique is to discriminate the samples in the feature space by either linear or nonlinear classifiers. Thus, it can be viewed as a typical clustering problem of pattern recognition. First, partition the image into regions or blocks and virtually create the corresponding coarse resolution lattice, as shown in Figure 1. Figure 1 depicts the multi-resolution pyramid that shows the relationship between the two resolution levels. Using the aforementioned methods, each pattern block at the measurement level corresponds to a certain number of features. Because of the links between the two resolution levels, every single pixel at the coarse resolution level can be represented by a vector whose elements are exactly the features generated from the corresponding pattern block at the fine resolution level. Repeating this procedure, groups of samples in a high dimensional feature space can be made. Figure 2 demonstrates the distribution of the samples that form three categories in a three dimensional space. The three axes stand for the three pre-selected features and the size of samples is equal to the number of partitions at the fine resolution level. The next step is to classify the samples with an appropriate clustering method [8]. After clustering the samples, the resulting segmentation image can be constructed block by block with all the pixels in a block being categorized into the same pattern. In addition to the features of SGLDM and GLDM, other specific statistics can also be used as potential features in this method [10].

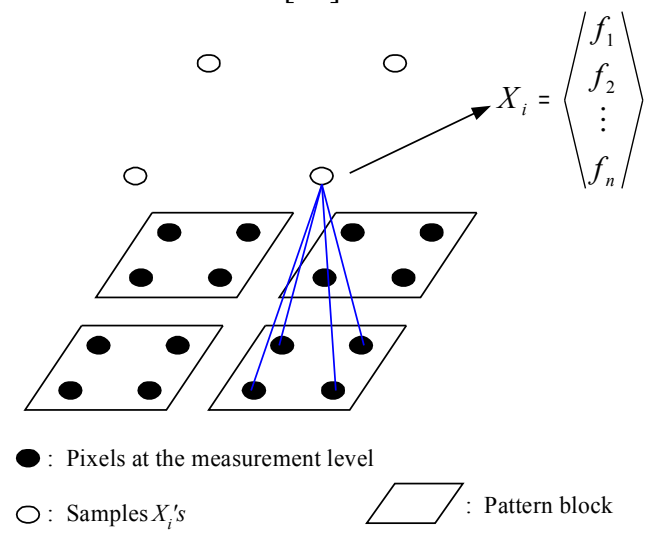

Figure 1. Multi-Resolution Pyramid

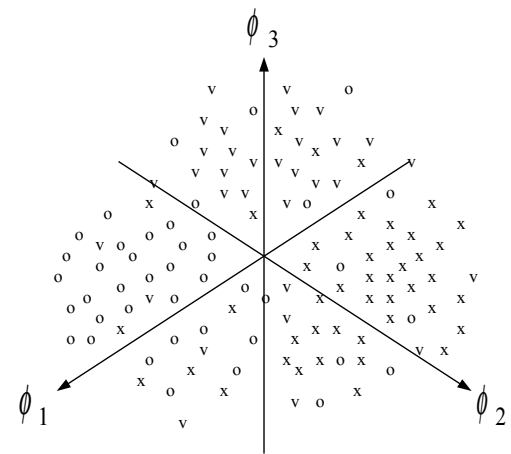

Figure 2. Distribution of Samples
In a word, the problem considered is a supervised image segmentation problem and the only supervised information is the number of features in an image. Through the multi-resolution pattern classification method, the coarse lattice is composed of feature vectors whose elements are the selected statistics. Application of a proper clustering algorithm is performed to classify the generated samples, followed by labeling the corresponding pixels at the fine resolution level based on the clustering result of the samples.

\section{CLUSTERING}

In the MPC method, parametric approaches are adopted for pattern clustering. The most widely used criteria are the class separability measures, which are shown as follows [8]:

$$
\begin{aligned}
& \text { 1. } J_{1}=\operatorname{tr}\left(S_{2}^{-1} S_{1}\right) \\
& \text { 2. } J_{2}=\log \left|S_{2}^{-1} S_{1}\right|
\end{aligned}
$$

where $S_{1}$ and $S_{2}$ can be $S_{b}, S_{w}$, or $S_{m}$. The matrices $S_{b}$, $S_{w}$, and $S_{m}$ are defined as:

- Within-class scatter matrix $S_{w}$ :

$$
S_{w}=\sum_{i=1}^{L} P_{i} \Sigma_{i} ;
$$

- Between-class scatter matrix $S_{b}$ :

$$
S_{b}=\sum_{i=1}^{L} P_{i}\left(M_{i}-M_{0}\right)\left(M_{i}-M_{0}\right)^{T} ;
$$

- $\quad$ Mixture scatter matrix $S_{m}$ :

$$
S_{m}=S_{w}+S_{b} .
$$

$M_{i}$ and $\Sigma_{i}$ are the mean matrix and the covariance matrix with respect to the $i^{\text {th }}$ category. $P_{i}$ 's are the probabilities for each class and $M_{0}=\sum_{i=1}^{L} P_{i} M_{i}$, where $L$ is the number of clusters. The class assignment is achieved by maximizing the class separability measure $J_{1}$, where $S_{1}$ and $S_{2}$ are assumed to be $S_{w}$ and $S_{m}$, respectively. Usually, the clustering algorithm is effective if the algorithm converges.

Without losing generality, assume that $M_{0}=0$ and $S_{m}=$ I. If the samples obtained do not satisfy these conditions, the coordinate origin can be shifted and the data can be whitened with respect to $S_{m}$. Notice that $S_{m}$ is generally full-ranked. Then the class separability becomes $J_{l}=J=\operatorname{tr}\left(S_{w}\right)$. Assume there are $N$ samples, $X_{1}, X_{2}, \ldots, X_{N}$, and each sample is to be classified to one of the $L$ classes, $\omega_{1}, \omega_{2}, \ldots, \omega_{L}$, i.e., the $i^{\text {th }}(i=1$ to $N)$ sample is to be categorized into the $k_{i}^{\text {th }}$ class denoted by $\omega_{k}(k=1$ to $L)$. The clustering criterion $J$ is a function of $\Omega$ and $\Gamma$, where $\Omega=\left[\omega_{1}\right.$, $\left.\omega_{2}, \ldots, \omega_{L}\right]^{T}$ and $\Gamma=\left[X_{1}, X_{2}, \ldots, X_{N}\right]^{T}$. The algorithm adopted is called the nearest mean reclassification rule (NNR), which is defined as follows:

1. Initially and randomly choose an initial classification, $\Omega(0)$ and calculate $M_{l}(0), \ldots$, $M_{L}(0)$, where $\Omega(0)$ and $M_{j}(0), j=1, \ldots, L$ are the first iteration of $\Omega$ and the class means. 
2. Let $N_{r}$ denote the number of samples in the $r^{\text {th }}$ class and the samples in the $r^{\text {th }}$ class are represented by $X_{j}^{(r)}, j=1, \ldots, N_{r}$. Then the criterion can be rewritten as:

$J=\operatorname{tr}\left(S_{w}\right)=\sum_{r=1}^{L} \frac{N_{r}}{N} \frac{1}{N_{r}} \sum_{j=1}^{N_{r}}\left(X_{j}^{(r)}-M_{r}\right)^{T}\left(X_{j}^{(r)}-M_{r}\right)=\frac{1}{N} \sum_{r=1}^{L} \sum_{j=1}^{N_{r}}\left\|X_{j}^{(r)}-M_{r}\right\|^{2}$ (1)

3. Change the cluster assignment of $X_{i}$ from the current class $k_{i}$ to class $j$ at the $m^{\text {th }}$ iteration. Also, remove the term $\left\|X_{i}-M_{k_{i}}(m)\right\|^{2}$ from the Eq. (1) and add a new term $\left\|X_{i}-M_{j}(m)\right\|^{2}$. Thus,

$$
\begin{gathered}
J(m)=J(m-1)+\Delta J(i, j, m), \\
\Delta J(i, j, m) \cong \frac{1}{N}\left\{\left\|X_{i}-M_{j}(m)\right\|^{2}-\left\|X_{i}-M_{k_{i}}(m)\right\|^{2}\right\} \text { (2) }
\end{gathered}
$$

Since the second term of Eq. (2) does not depend on $J$, the reclassification of $X_{i}$ at the $m^{\text {th }}$ iteration can be achieved by

$$
\begin{gathered}
j=\arg \min _{t}\left\|X_{i}-M_{t}(m)\right\|, \\
X_{i} \rightarrow \omega_{j}
\end{gathered}
$$

In the other words, calculate sample mean vectors $M_{l}(m), \ldots, M_{L}(m)$ at the $m^{\text {th }}$ iteration, and reclassify each $X_{i}$ according to the nearest $M_{j}$.

4. If the reclassification of any $X_{i}$ occurs, then calculate the new sample mean vectors $M_{1}(m+1), \ldots, \quad M_{L}(m+1)$ for the new class Remarks: assignment, and repeat Step 3. Otherwise stop.

1. The assumption about the nonsigularity of $S_{m}$ can be generally satisfied by carefully adopting the features. To avoid numerical illconditions of the resulting mixture covariance matrix, the estimated features can be normalized to have the same level of values. For instance, for each partition the feature $f_{i}$ can be replaced by $f_{i}^{\prime}$, where $f_{i}^{\prime} \cong f_{i} / \max \left[f_{i}\right]$.

2. Only the means contribute to the determination of the classification boundary. Covariance matrices do not affect the boundary. Apparently, clusters are divided by piecewise linear bisectors. This is just equivalent to applying a linear classifier to the created samples.

3. The initial random class assignment does not impose any extra constraints and instability on the algorithm.

4. The iterative NNR algorithm does not guarantee convergence. Also, the process may be trapped at a local minimum point and fails to get a global minimum [11]. However, except these potentially uneven factors, this approach works well in general.

One of the major reasons of choosing the NNR algorithm is that the number of categories is necessary to be pre-assigned. As mentioned before, the supervised techniques were used for image segmentation. Thus, it is quite natural to treat NNR as the fundamental linear classifier.
In this section, the multi-resolution pattern classification (MPC) method will be applied to rust images, in which the rust spots or areas need to be discriminated from the background such as the surfaces of bridges. Basically, the quality of the acquired images, usually color ones, has a deep influence on the segmentation performance. Unfortunately, rust images sometimes contain reflected light or shadows. The basic nature of images can be characterized by two components: the illumination and the reflectance. This illuminationreflectance model can be used as the basis for a frequency domain processing, which is useful for improving image quality by simultaneous brightness range compression and contrast enhancement [12]. Before applying MPC to any rust images, to overcome the effect of non-uniform illumination, a technique called "homomorphic filtering" will be used to pre-process the color rust images [12].

\subsection{Homomorphic Filtering}

An image denoted by $f(x, y)$ can be expressed in terms of its illumination $i(x, y)$ and reflectance $r(x, y)$ as

$$
f(x, y)=i(x, y) r(x, y) \text {. }
$$

The illumination component of an image is generally characterized by slow spatial variations, while the reflectance component tends to vary abruptly. These characteristics are particularly useful in separating these two components in the frequency domain. Assume

then

$$
z(x, y)=\log f(x, y),
$$

$$
Z(u, v)=I(u, v)+R(u, v)
$$

where $I(u, v)$ and $R(u, v)$ are the Fourier transforms of $\log i(x, y)$ and $\log r(x, y)$, respectively. Since the characteristics of the illumination and the reflectance distinguish the low and the high frequencies of $Z(u, v)$, image enhancement (especially for nonuniformly illuminated images) can be achieved by applying a homomorphic filter $H(u, v)$, which could adjust the low- and the high-frequency components differently. Typically, the filter tends to decrease the low frequencies and amplify the high frequencies. The net result is a combined dynamic range compression and contrast enhancement. The homomorphic filtering approach is expressed in Figure 3.

Generally, $H(u, v)$ is a circularly symmetric filter. Figure 4 depicts the typical shapes of $H(u, v)$ and its cross section.

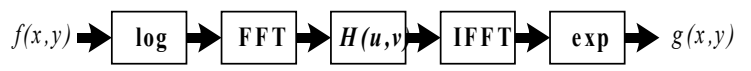

Figure 3. Homomorphic Filtering Approach

\section{DISCRIMINATION OF RUST IMAGES}




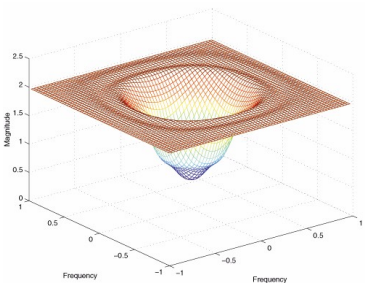

(a)

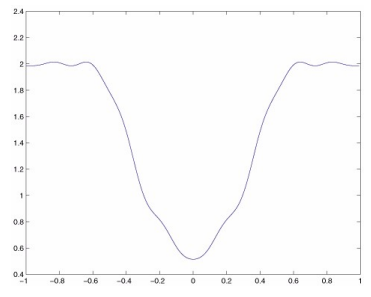

(b)

Figure 4. Typical Shape of Homomorphic Filter $H(u, v):$ (a) frequency response,

(b) cross section of frequency response

Figure 5 shows six color rust images. Figure 5 (c), (d), (f) are affected by non-uniform illumination and with low contrast. Before applying MPC to segment rust and background, homomorphic filtering technique was utilized to pre-process these inferior images and the filtered images are demonstrated in Figure 6.

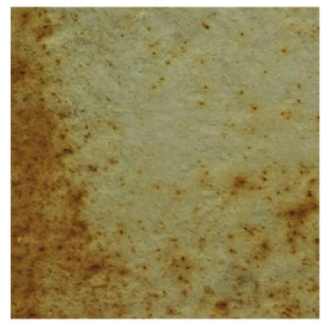

(a)

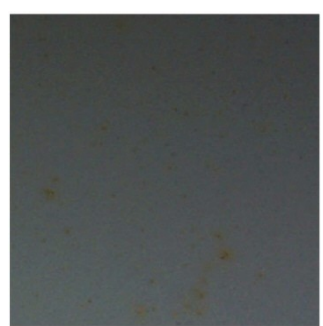

(c)

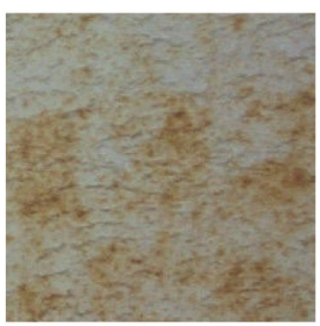

(e)

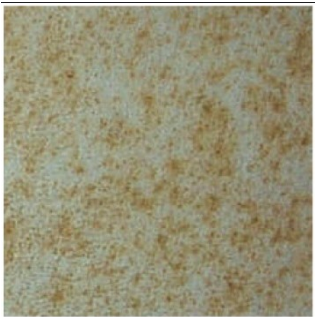

(b)

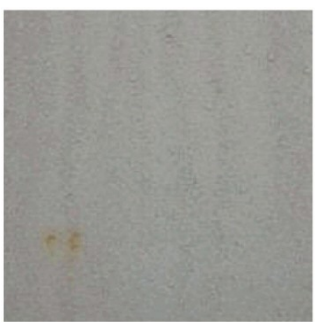

(d)

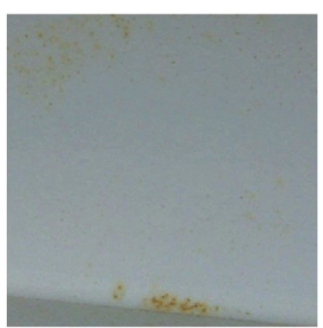

(f)

Figure 5. Color Rust Images

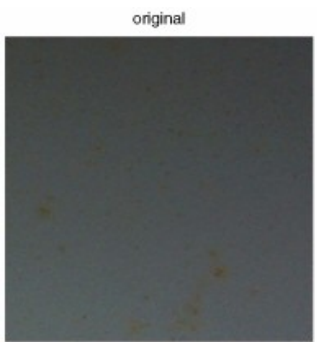

original

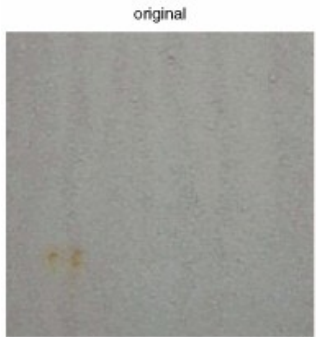

oniginal

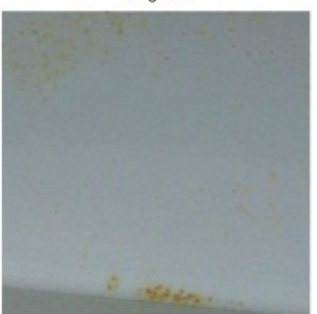

(d)

c)

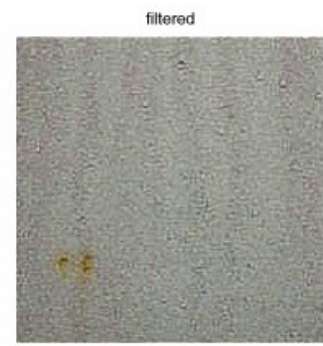

filtered

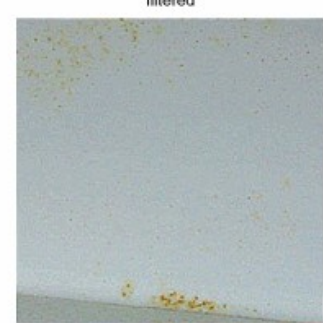

(f)

Figure 6. Original and Filtered Rust Images

\subsection{Segmentation of Rust Images}

After homomorphically filtering the rust images, the original color images are converted into gray level images. At this stage, the goal is to differentiate the rust from the background by extracting the rust features using the MPC algorithm. To enhance the resolution of segmentation images, the partition size is set to be 4-by-4. The underlying statistics of MPC for each example are MEAN, VAR, and the one listed in Table 1, where MEAN and VAR stand for the mean and the variance of the corresponding pattern block. The underlying number of gray levels is 32. The average processing time is 60 seconds. Figure 7 and Figure 8 demonstrate the resulting segmented images. The results indicated the MPC method is effective. As for the rust image shown in Figure 5(c), the seriously non-uniform illumination lessens the local contrast in the shadow area. Even after homomorphic filtering, the quality of the local contrast is not well improved, as shown in Figure 6(c).

Table 1. Statistics Used for the MPC method

\begin{tabular}{|c|c|c|c|c|c|c|}
\hline Figure 5: & $(a)$ & $(b)$ & $(c)$ & $(d)$ & $(e)$ & $(f)$ \\
\hline Statistics & $\begin{array}{c}C O R \\
(S G L D M)\end{array}$ & $\begin{array}{c}C O R \\
(S G L D M)\end{array}$ & $\begin{array}{c}C O R \\
(S G L D M)\end{array}$ & $\begin{array}{c}C O R \\
(G L D M)\end{array}$ & $\begin{array}{c}C O R \\
(S G L D M)\end{array}$ & $\begin{array}{c}C O R \\
(S G L D M)\end{array}$ \\
\hline
\end{tabular}




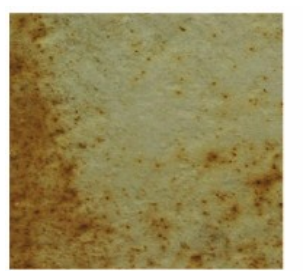

(a)
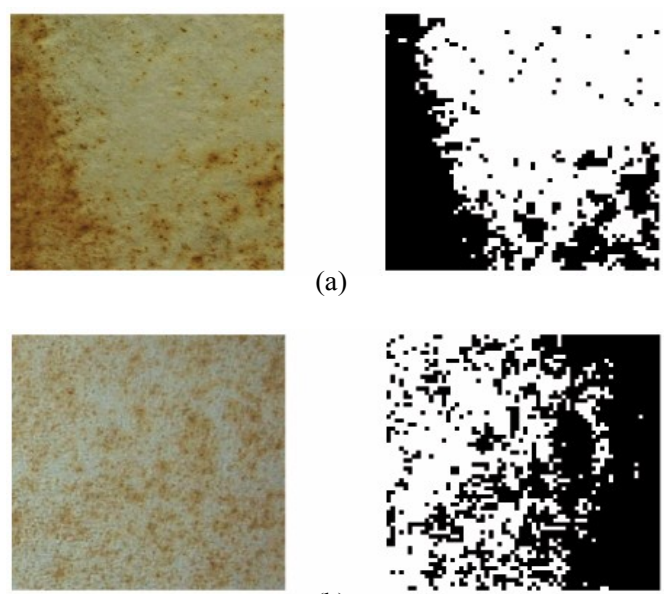

(b)
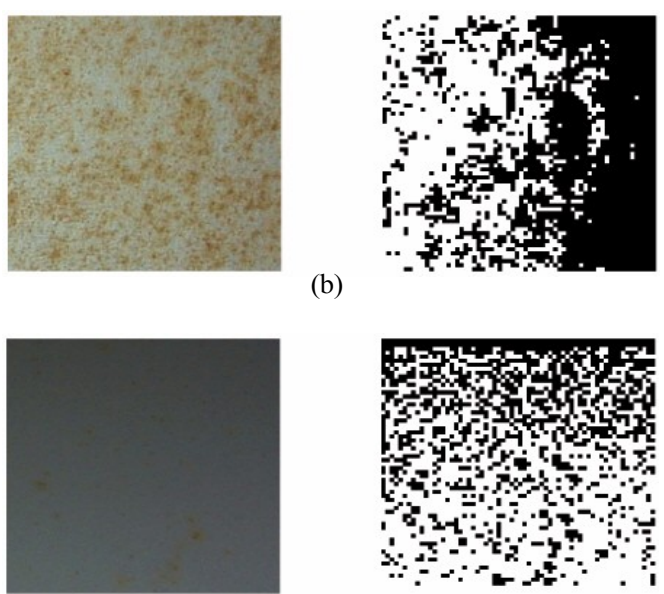

(c)

Figure 7. Original and Segmented Rust Images ((a) - (c))

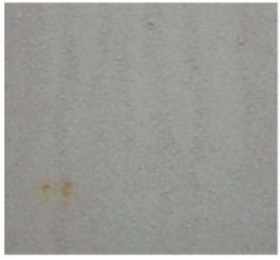

(d)
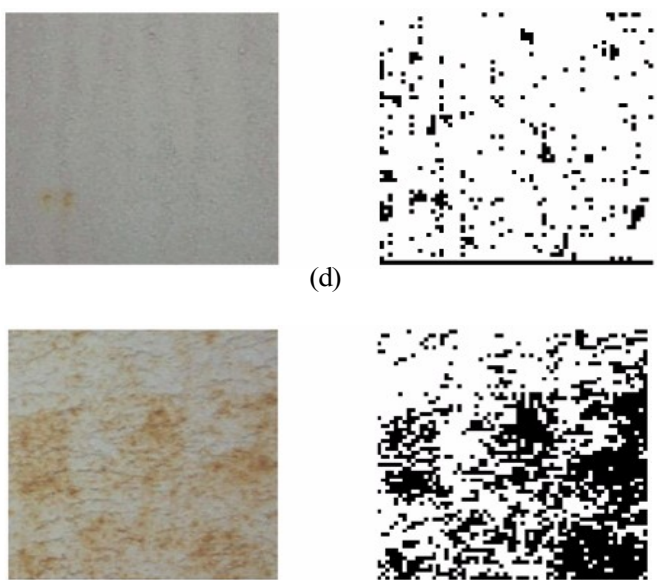

(e)

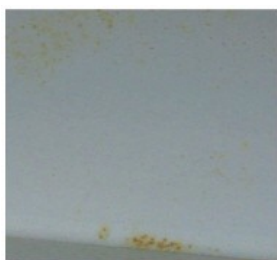

(f)
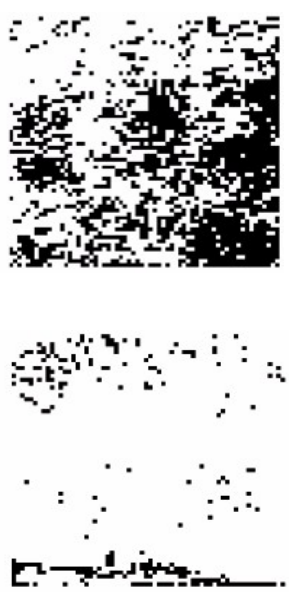

Figure 8. Original and Segmented Rust Images $((d)-(f))$

\section{CONCLUSIONS}

In this paper, the multi-resolution pattern classification (MPC) method was presented. The major contribution of this method was the combination of the multi-resolution pyramid image model and the pattern discrimination techniques in a high dimensional feature space. Experiments showed that this method has effectively reduced the computational costs and successfully segmented images. In addition, the homomorphic filtering technique was introduced to adjust the illumination and the reflectance of non-uniformly illuminated images.

\section{REFERENCES}

[1] Haralick, R. M., "Statistical and Structural Approaches to Textures," IEEE Proceedings, Vol. 67, No. 5, pp. 786-804, May 1979.

[2] Reed, T. R. and Hans Du Buf, J. M., "A Review of Recent Texture Segmentation and Feature Extraction Techniques," CVGIP-Image Understanding, Vol. 57, No. 3, pp. 359-372, May 1993.

[3] Conners, R. W. and Harlow, C. A., "A Theoretical Comparison of Texture Algorithm," IEEE Trans Pattern Anal. Machine Intell., Vol. PAMI-2, No. 3, pp. 204-222, May 1980.

[4] Chang, Y. C., "Statistical Models for MRF Image Restoration and Segmentation," Ph.D. Dissertation, Purdue University, West Lafayette, Indiana, USA, August 2000.

[5] Unser, M., and Eden, M., "Multi-resolution Feature Extraction and Selection for Texture Segmentation," IEEE Trans Pattern Anal. Machine Intell., Vol. 11, No. 7, pp. 717-728, July 1989.

[6] Hong, T. H., Narayanan, K. A., Peleg, S., Rosenfeld, A., and Silberberg, T., "Image Smoothing and Segmentation by Multi-resolution Pixel Linking: Further Experiments and Extensions," IEEE Trans Syst., Man, and Cybern., Vol. SMC-12, No. 5, pp. 611-622, September 1982.

[7] Ng, I., Kittler, J., and Illingworth, J., "Supervised Segmentation Using a Multi-resolution Data Representation," Signal Processing, Vol. 31, No. 2, pp. 133-163, March 1993.

[8] Fukunaga, K., Introduction to Statistical Pattern Recognition, $2^{\text {nd }}$ Edition, Academic Press, 1990.

[9] Weszka, J. S., Dyer, C. R., and Rosenfeld, A, “A Comparative Study of Texture Measures for Terrain Classification," IEEE Trans Pattern Anal. Machine Intell., Vol. SMC-6, No. 3, pp. 269-285, April 1976.

[10] Geman, D., Geman, S., Graffigne, C., and Dong, P., "Boundary Detection by Constrained Optimization," IEEE Trans Pattern Anal. Machine Intell., Vol. 12, No. 7, pp. 609-627, July 1990.

[11] Fukunaga, K., and Koontz, W. L. G., "A Criterion and an Algorithm for Grouping Data," IEEE Trans Computers, Vol. C-19, No. 5, pp. 917923, 1970.

[12] Gonzalez, R. C., and Woods, R. E., Digital Image Processing, Addison-Wesley Publishing Company, Inc., 1992. 